\title{
Bandwidth Allocation for a Revenue-Aware Network Utility Maximization
}

\author{
David Mayer and Javier A. Barria, Member, IEEE
}

\begin{abstract}
In this letter, we consider the Network Utility Maximization (NUM) problem in which a loss-related revenue indicator is set up by an operator as a constraint. We propose an algorithm which artificially modifies capacity constraints of a standard NUM algorithm. This results in a price-based mechanism that drives users into an operating point which maximizes total user utility for a given revenue indicator constraint.
\end{abstract}

Index Terms-Bandwidth allocation, network utility maximization, revenue optimization, loss network.

\section{INTRODUCTION}

B ANDWIDTH allocation has been extensively studied with various objectives in view, especially objectives concerning the users (e.g. utility maximization and end-toend delay minimization) and objectives of the operator's interest (e.g. revenue maximization and cost minimization). The question of combining the users' and operators' concerns remains an open issue. The motivation for this work stems from the fact that selfish user behavior comes at high cost for the operator and is conflicting with Traffic Engineering efforts [1].

In this letter, we attempt to solve the problem of Network Utility Maximization (NUM) with a loss-related constraint set up by the operator. Traditional NUM algorithms [2][3] result in fully utilized links. Assuming that users only pay for successfully delivered traffic, the loss associated with fully utilized links reduces operator's revenue. Hence we choose as a loss-related metric an operator's revenue indicator and solve the problem of NUM with this revenue indicator used as a constraint. To our knowledge, there is not a published work on trade-offs between utility and revenue in loss networks. However, several papers exist on optimization involving both revenue and some aspect of utility, typically fairness (e.g. [4]).

Our approach draws from the work of Mitra [5] on loss networks and uses a price-based mechanism to control user demand [2].

\section{Model And Problem Formulation}

Consider a set $\mathcal{L}$ of $L$ links and a set $\mathcal{F}$ of $F$ flows. Associated with each link $l \in \mathcal{L}$ there is a loss function $L_{l}\left(x_{l}\right)$, where $x_{l}$ is the flow on link $l$. Link $l$ capacity is denoted by $C_{l}$. Flow configuration is given and represented by routing matrix $\mathbf{A}=\left(a_{i, l} ; i \in \mathcal{F}, l \in \mathcal{L}\right)$, where $a_{i, l}=1$ if flow $i$

Manuscript received February 23, 2007. The associate editor coordinating the review of this letter and approving it for publication was Dr. Aleksandra Smiljanic. The work of D. Mayer was supported by the UK Engineering and Physical Sciences Research Council under Grant GR/S52360/01.

The authors are with the Department of Electrical and Electronic Engineering, Imperial College London, London SW7 2AZ, UK (e-mail: \{david.mayer, j.barria\}@imperial.ac.uk).

Digital Object Identifier 10.1109/LCOMM.2007.070280. uses link $l$ and $a_{i, l}=0$ otherwise. Each flow $i$ uses one path denoted by $R_{i}=\left\{l \in \mathcal{L} \mid \mathbf{A}_{i, l}=1\right\}$. Flow $i \in \mathcal{F}$ is associated with a user $i$ and a utility function $U_{i}\left(f_{i}\right)$, where $f_{i}$ is the bandwidth demand of user $i$. Let $\mathbf{f}=\left(f_{i}, i \in \mathcal{F}\right)$. We assume that utility functions are concave increasing.

Our goal is to optimize the capacity constraints provided to the standard NUM algorithm ([2][3]) in order to maximise total user utility $U$ achieved by the NUM algorithm subject to the operator's revenue indicator $\pi$ being equal to or greater than a positive constant $\pi_{m i n}$. The optimization problem of the operator is $\pi$-NUM:

$$
\begin{array}{ll} 
& \max _{\mathbf{k} \geq 0} U(\mathbf{k}) \\
\text { s.t. } & \pi_{\min }-\pi(\mathbf{k}) \leq 0,
\end{array}
$$

where $\mathbf{k}=\left(k_{1}, \ldots, k_{L}\right)$ is the control variable and $U(\mathbf{k})$ is the maximum of the following utility maximising problem. $\operatorname{NUM}(\mathbf{k})$ :

$$
\begin{array}{ll} 
& U(\mathbf{k})=\max _{\mathbf{f} \geq 0} \sum_{i \in \mathcal{F}} U_{i}\left(f_{i}\right) \\
\text { s.t. } & \sum_{i \in \mathcal{F}}\left(f_{i} a_{i, l}-k_{l} C_{l}\right) \leq 0, \quad \forall l \in \mathcal{L}, k_{l} \in \mathbf{k} .
\end{array}
$$

Denote $\mathbf{f}^{*}(\mathbf{k})$ the maximiser of $\operatorname{NUM}(\mathbf{k})$. Vector $\mathbf{k}$ modifies the capacity constraints. Once found, the standard distributed NUM algorithm can be run, realizing thus (1). Associated with the solution of (2) (and obtained via the distributed algorithm), is a vector of Lagrange multipliers $\boldsymbol{\lambda}(\mathbf{k})=\left(\lambda_{l}(\mathbf{k}), l \in \mathcal{L}\right)$. Using a price-based control of user demand framework, we assume that users maximise their surplus. Hence if for flow $i$ the price per unit of bandwidth is $p_{i}$, the user will choose demand $f_{i}$, such that

$$
f_{i}=\arg \max _{f}\left\{U_{i}(f)-p_{i} f\right\} .
$$

In this setting, and for concave utility functions, surplus is maximized at a point where marginal utility equals price. When the price seen by flow $i$ is given by the sum of Lagrange multipliers $\boldsymbol{\lambda}(\mathbf{k})$ over all links on path $R_{i}$,

$$
p_{i}(\mathbf{k})=\sum_{l \in R_{i}} \lambda_{l}(\mathbf{k}),
$$

then the solution $f_{i}$ found by each user corresponds to the solution $f_{i}^{*}(\mathbf{k})$ of $\operatorname{NUM}(\mathbf{k})$ [3]. The value of the revenue indicator $\pi$ (referred to as revenue from now on) is calculated as if the operator charged prices $p_{i}(\mathbf{k})$ for successfully carried traffic,

$$
\pi(\mathbf{k})=\sum_{i \in \mathcal{F}} p_{i}(\mathbf{k}) f_{i}^{*}(\mathbf{k})\left(1-L^{(i)}\right),
$$

where $L^{(i)}$ denotes the loss encountered by flow $i$ along its path. 
We use a single-server queue with a buffer of size $K$ to model link loss in the network. Note that our algorithm is not dependent on the specific loss function. We give numerical results for both small and large buffer, accounting for different loss behavior. We assume that packets arrive to link $l$ according to a Poisson process with mean rate $x_{l}$ and the packet length is exponentially distributed with mean $s$. Service rate is then exponentially distributed with mean $\mu_{l}=C_{l} / s$. This is an $\mathrm{M} / \mathrm{M} / 1 / \mathrm{K}$ queue, for which the loss probability is defined as $L_{l}\left(x_{l}\right)=\rho_{l}^{K} \frac{1-\rho_{l}}{1-\rho_{l}^{K+1}}$, where $\rho_{l}=\frac{x_{l}}{\mu_{l}}=\frac{x_{l}}{C_{l}} s$ is the offered load. Furthermore, assuming link independence, the loss encountered by flow $i$ can be calculated as $L^{(i)} \approx$ $1-\prod_{l \in R_{i}}\left(1-L_{l}\right)$, where $L_{l}, l \in \mathcal{L}$ is obtained from a system of fixed-point equations as in [6].

\section{Proposed Solution}

Problem (1) has a concave objective function, but nonconvex constraints (revenue (5) is a non-monotonic function of demand) for which a suitable optimization method is that of Augmented Lagrangians [7]. This is essentially a penalty method, which converts constrained problems into a sequence of unconstrained problems $Q_{\gamma_{t}}(\cdot), t=0,1, \ldots$ by adding a penalty function $P_{\gamma}(\cdot)$ and an increasing penalty factor $\gamma$. The solution of $Q_{\gamma_{t}}(\cdot)$ is used as the starting point for solving $Q_{\gamma_{t+1}}(\cdot)$. This method does not require a feasible initial point and is well-suited for nonlinear constraints. ${ }^{1}$ The augmented Lagrangian function is

$$
Q_{\gamma}(\mathbf{k}, \mu)=U(\mathbf{k})-P_{\gamma}(\mathbf{k}, \mu) .
$$

The inclusion of Lagrange multiplier $\mu$ within the penalty function enables convergence without the penalty factor $\gamma \rightarrow$ $\infty .^{2}$ We use the following penalty (adopted from [7])

$$
P_{\gamma}(\mathbf{k}, \mu)=\frac{1}{2 \gamma}\left([\max \{0, \mu+\gamma g(\mathbf{k})\}]^{2}-\mu^{2}\right),
$$

where $g(\mathbf{k})=\pi_{\min }-\pi(\mathbf{k})$. The maximization of the unconstrained Lagrangian (6) requires the sensitivity of total utility and revenue generated by the solution of (2) with respect to capacity multipliers $\mathbf{k}$. From the theory of Lagrange multipliers it is known that sensitivity of the objective function with respect to constraints equals the corresponding Lagrange multipliers, here $\frac{\partial U}{\partial C_{j}}=\lambda_{j}$, hence $\frac{\partial U}{\partial k_{j}}=C_{j} \lambda_{j}$. Note that the sensitivity of revenue $\frac{\partial \pi}{\partial k_{j}}$ has to be replaced by a finite difference $\frac{\Delta \pi}{\Delta k_{j}}$ due to discontinuities of Lagrangians caused by the change of the set of active constraints in (2). Sensitivity of revenue is derived below by extending the results in [5] for interdependent demand and price.

The proposed optimization consists of repeated increases of the augmented Lagrangian (6) by shifting vector $\mathrm{k}$ in the steepest ascent direction,

$$
\mathbf{k}_{t+1}=\mathbf{k}_{t}+\alpha \cdot \nabla Q_{\gamma}\left(\mathbf{k}_{t}, \mu_{t}\right),
$$

\footnotetext{
${ }^{1}$ Convergence to global maximum requires that in each iteration a global maximum of $Q(\cdot)$ is found. We rely on the initial point lying in proximity of the global maximum. This is in practice, given by common packet loss functions, $\mathbf{k}=1$, i.e. the point corresponding to the original link capacities.

${ }^{2}$ The idea is to increase the penalty factor to approach feasible range and then to adjust only the Lagrangian multiplier in the near-optimum range, preventing $\gamma \rightarrow \infty$.
}

where $\alpha$ is a decreasing step size. The gradient of (6) at iteration $t$ is

$$
\begin{aligned}
\nabla Q_{\gamma_{t}}\left(\mathbf{k}_{t}, \mu_{t}\right)= & \mathbf{C} \cdot \lambda\left(\mathbf{k}_{t}\right)+ \\
& \max \left\{0, \mu_{t}+\gamma_{t} g\left(\mathbf{k}_{t}\right)\right\} \cdot \frac{\Delta \pi\left(\mathbf{k}_{t}\right)}{\Delta k} .
\end{aligned}
$$

Penalty factor $\gamma$ and multiplier $\mu$ are updated as in [7, p.123]:

$$
\begin{aligned}
\mu_{t+1} & =\max \left\{0, \mu_{t}+\gamma_{t}\left(\pi_{\min }-\pi\left(\mathbf{k}_{t}\right)\right)\right\} \\
\gamma_{t+1} & = \begin{cases}\beta \gamma_{t} & \text { if } \frac{\left|\pi_{\min }-\pi\left(\mathbf{k}_{t}\right)\right|}{\left|\pi_{\min }-\pi\left(\mathbf{k}_{t-1}\right)\right|} \geq \delta \\
\gamma_{t} & \text { otherwise. }\end{cases}
\end{aligned}
$$

Scalar $\beta$ is typically chosen $\beta \in[1.1,2]$ and $\delta=0.75$. The algorithm terminates when the following condition holds:

$$
\left|\pi_{\min }-\pi\right| \leq \epsilon_{\pi} \wedge\left\|\nabla Q_{\gamma_{t}}\left(\mathbf{k}_{t}, \mu_{t}\right)\right\| \leq \epsilon_{P}
$$

where typically $\epsilon_{\pi}=0.01$ and $\epsilon_{P}=1$. The pseudocode of the proposed algorithm for solving (1) is shown in Table I.

\section{Sensitivity of revenue to capacity constraints}

In order to update the capacity multipliers vector $\mathbf{k}$ in (8) we need to obtain the sensitivity of revenue with respect to $\mathbf{k}, \frac{\Delta \pi}{\Delta k}=\left(\frac{\Delta \pi}{\Delta k_{j}}, j=1,2, \ldots, L\right)$. Let us write revenue (5) as a function of demand and price, $\pi=y(\mathbf{f}, \mathbf{p})$. First, we derive the total derivative with respect to demand $f_{i}$,

$$
\frac{\mathrm{d} \pi}{\mathrm{d} f_{i}}=\frac{\partial \pi}{\partial f_{i}}+\frac{\partial \pi}{\partial p_{i}} \frac{\partial p_{i}}{\partial f_{i}}
$$

Revenue sensitivity is known [5] and can be calculated as $\frac{\partial \pi}{\partial f_{i}}=\left(1-L^{(i)}\right)\left(p_{i}-\sum_{l \in R_{i}} c_{i l}\right)$, where $c_{i l}$ is the implied cost of flow $i$ on link $l$ calculated using the expected buffer occupancy of an M/M/1/K queue. From revenue definition (5) we have $\frac{\partial \pi}{\partial p_{i}}=f_{i}\left(1-L^{(i)}\right)$. From (3) we have $p_{i}=U_{i}{ }^{\prime}$, hence $\frac{\partial p_{i}}{\partial f_{i}}=U_{i}^{\prime \prime}\left(f_{i}\right)$. Then (13) can be expressed as

$$
\frac{\mathrm{d} \pi}{\mathrm{d} f_{i}}=\left(1-L^{(i)}\right)\left(p_{i}-\sum_{l \in R_{i}} c_{i l}+f_{i} U_{i}^{\prime \prime}\left(f_{i}\right)\right) .
$$

The term $f_{i} U_{i}^{\prime \prime}\left(f_{i}\right)$ is negative and accounts for the dependency of price and demand. We use (14) in the calculation of the revenue sensitivity, which is given by the following total finite difference

$$
\frac{\Delta \pi}{\Delta k_{j}}=C_{j} \frac{\Delta \pi}{\Delta C_{j}}=C_{j} \sum_{i \in \mathcal{F}} \frac{\mathrm{d} \pi}{\mathrm{d} f_{i}} \frac{\Delta f_{i}}{\Delta C_{j}} .
$$

\section{Sensitivity of demand to capacity constraints}

The remaining unknown in (15) is the change of demand $\Delta f_{i}$ upon adding a small $\Delta C$ to link $j$ in (2), $\frac{\Delta f_{i}}{\Delta C_{j}}$, which we approximate as follows. Total utility $U$ is given by a sum of individual utilities, hence it can be approximated by a sum of Taylor series approximations, here of second order:

$$
U\left(\mathbf{f}^{*}+\Delta \mathbf{f}\right) \approx U\left(\mathbf{f}^{*}\right)+\sum_{i \in \mathcal{F}} U_{i}^{\prime} \Delta f_{i}+\sum_{i \in \mathcal{F}} \frac{1}{2} U_{i}^{\prime \prime}\left(\Delta f_{i}\right)^{2},
$$

where $\mathbf{f}^{*}$ is the current utility maximising demand vector and $\Delta \mathbf{f}$ is the vector of demand changes. Vector $\Delta_{j} \mathbf{f}^{*}$ of optimal changes upon adding small $\Delta C$ to link $j$ is approximated by the solution of the following optimization:

$$
\begin{array}{ll}
\max _{\boldsymbol{\Delta}_{j} \mathbf{f}} & \sum_{i \in \mathcal{F}}\left[U_{i}^{\prime}\left(f_{i}^{*}\right) \Delta_{j} f_{i}+\frac{1}{2} U_{i}^{\prime \prime}\left(f_{i}^{*}\right)\left(\Delta_{j} f_{i}\right)^{2}\right] \\
\text { s.t. } & x_{l}+\sum_{i \in \mathcal{F}} \Delta f_{i} \cdot a_{i, l} \leq\left\{\begin{array}{l}
C_{l}, l \in \mathcal{L} \backslash j \\
C_{l}+\Delta C, l=j
\end{array}\right.
\end{array}
$$


TABLE I

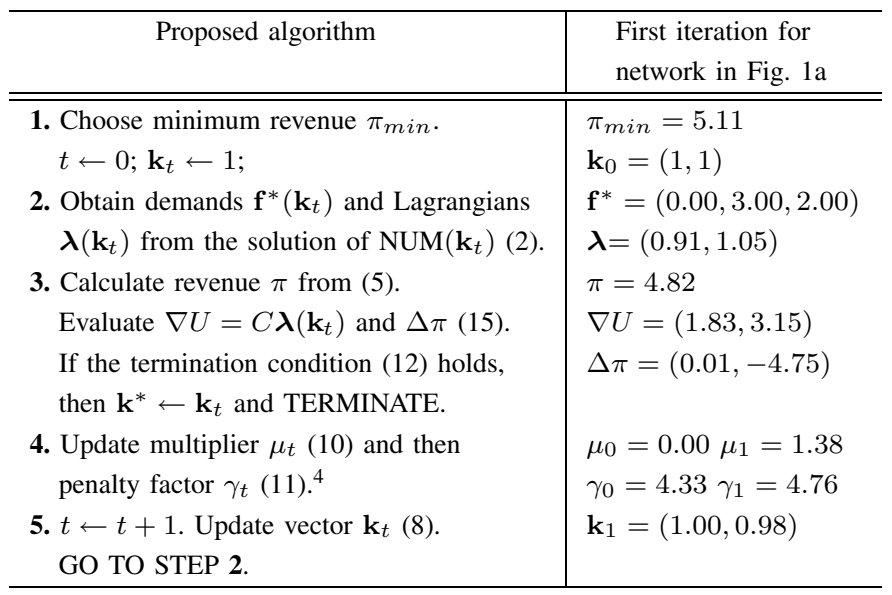

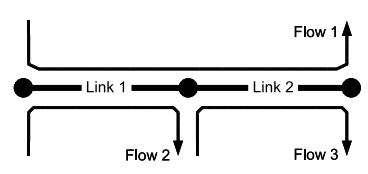

a)

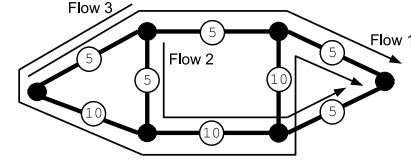

b)
Fig. 1. Networks used in the numerical examples. Circles in network b) specify link capacities.

The rationale for this optimization is that the sum of utility changes $\sum_{i \in \mathcal{F}} \Delta U_{i}$ approximated by the last two terms in (16) must be maximal and that link flow must not exceed any link capacity except for the augmented link $j$. This optimization has the standard form of a quadratic objective function $\boldsymbol{\Delta}_{j} \mathbf{f} \mathbf{y}+\frac{1}{2} \boldsymbol{\Delta}_{j} \mathbf{f} \mathbf{H}\left(\boldsymbol{\Delta}_{j} \mathbf{f}\right)^{T}$ where, in our case, $\mathbf{y}=\left(U_{1}^{\prime}\left(f_{1}^{*}\right), \ldots, U_{F}^{\prime}\left(f_{F}^{*}\right)\right)^{T}, \mathbf{H}$ is a diagonal matrix of second derivations with $H(i, i)=U_{i}^{\prime \prime}\left(f_{i}^{*}\right)$ and so (17) can be easily solved using standard algorithms (e.g. [7]). Solution $\Delta_{j} \mathbf{f}^{*}$ of (17) is used to approximate $\frac{\Delta f_{i}}{\Delta C_{j}}$ in (15).

\section{Numerical Results}

First we show the proposed procedure on a 2-link network accommodating 3 users (see Fig. 1a). Utility functions of the users are in the form $U_{i}\left(f_{i}\right)=a_{i}\left(1-2^{-b_{i} f_{i}}\right)$ and parameters of the problem are: $a_{1}=2, a_{2}=5, a_{3}=10, b_{1}=b_{2}=b_{3}=$ $0.8, C_{1}=2, C_{2}=3$. Solutions for various revenue constraints are shown in Fig. 2, where axes represent elements of vector k. Contours of revenue and total utility were obtained by exhaustive search. All solutions were reached starting from $\mathbf{k}_{0}=(1,1)$, although in practice previous solutions could serve as a starting point. Table I shows the data for the first iteration of the algorithm for $\pi_{\min }=5.11$. Fig. 3a shows the revenue-utility trade-off for various buffer size to demonstrate the dependence of the trade-off on loss characteristics of the system. ${ }^{3}$ Results for a larger 8-link network (see Fig. 1b) are shown in Fig. $3 b$.

\section{FinAl REMARKS}

This letter addresses network utility maximization with a constraint on an operator's revenue indicator and proposes an

\footnotetext{
${ }^{3}$ Large buffer size corresponds to effective bandwidth close to the average rate (small loss), whereas small buffer size models effective bandwidth close to the peak rate (high loss).

${ }^{4}$ Multipliers are initialized as $\mu_{0}=0$ and $\gamma_{0}=\left|\frac{\|\nabla U\|}{\|\nabla \pi\| \cdot\left(\pi_{m i n}-\pi\right)}\right|$.
}

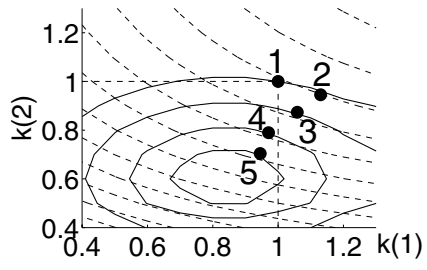

\begin{tabular}{ccc}
$m$ & $\pi$ & $\Sigma U$ \\
\hline 1 & 4.82 & 11.46 \\
2 & 4.86 & 11.50 \\
3 & 5.11 & 11.12 \\
4 & 5.31 & 10.61 \\
5 & 5.43 & 10.15
\end{tabular}

Fig. 2. Contours of revenue (full line) and utility (dashed line) for network in Fig. 1a. Circles show solutions for particular revenue constraints $\pi_{\min }$ indexed by $m$. Solutions were reached individually from starting point $\mathbf{k}_{0}=$ $(1,1)$ using the proposed algorithm and for buffer size $K=24$.
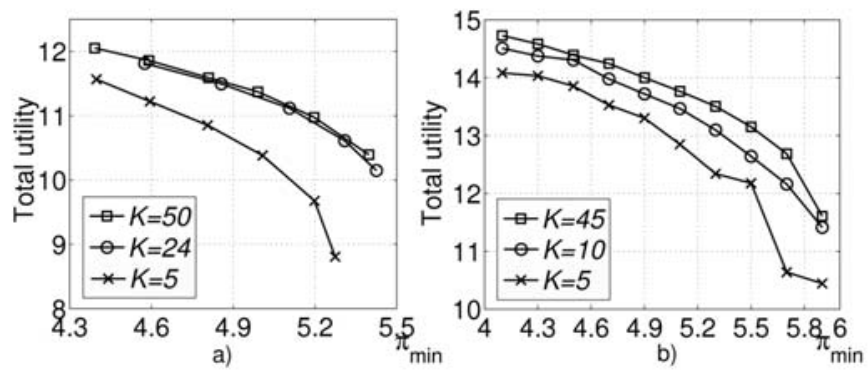

Fig. 3. Total utility as a function of minimum revenue constraint for different buffer sizes $K$ as obtained by the proposed algorithm for network in Fig. 1a (a) and Fig. $1 \mathrm{~b}(\mathrm{~b})$.

algorithm for controlling this trade-off. Although some parameters of the proposed algorithm are computed centrally (the revenue sensitivity vector), once they are communicated to network nodes, the NUM algorithm runs in a fully distributed way. At present we are developing the distributed version of this framework which will be reported in a forthcoming paper.

Regarding the assumption of concave utility functions, recent work on maximization of non-concave utility functions offers new methods based on the standard NUM algorithm. We are therefore also extending our work in this direction.

\section{REFERENCES}

[1] L. Qiu, Y. R. Yang, Y. Zhang, and S. Shenker, "On selfish routing in Internet-like environments," in Proc. SIGCOMM03, Karlsruhe, Germany, 2003.

[2] F. Kelly, A. Maulloo, and D. Tan, "Rate control in communication networks: shadow prices, proportional fairness and stability," J. Operational Research Society, vol. 49, 1998.

[3] S. Low and D. Lapsely, "Optimization flow control. I. Basic algorithm and convergence," IEEE/ACM Trans. Networking, vol. 7, no. 6, pp. 861874, 1999 .

[4] M. Zukerman, L. Tan, H. Wang, and I. Ouveysi, "Efficiency-fairness tradeoff in telecommunications networks," IEEE Commun. Lett., vol. 9, no. 7, pp. 643-645, 2005.

[5] D. Mitra, J. Morrison, and K. Ramakrishnan, "ATM network design and optimization: a multirate loss network framework," IEEE/ACM Trans. Networking, vol. 4, no. 4, pp. 531-543, 1996.

[6] F. Kelly, "Loss networks," The Annals of Applied Probability, vol. 1, no. 3, pp. 319-378, 1991.

[7] D. P. Bertsekas, Constrained Optimization and Lagrange Multiplier Methods. Academic Press, INC. (LONDON) LTD., 1982. 